Carnets de géographes

GÉOGRAPHES

5 | 2013

Géographie humanimale

\title{
Matérialisations du souvenir en montagne les enjeux identitaires des places et des placements
}

\section{Emmanuelle Petit}

\section{(2) OpenEdition}

\section{Journals}

Édition électronique

URL : http://journals.openedition.org/cdg/1111

DOI : $10.4000 / c d g .1111$

ISSN : 2107-7266

Éditeur

UMR 245 - CESSMA

\section{Référence électronique}

Emmanuelle Petit, « Matérialisations du souvenir en montagne les enjeux identitaires des places et des placements », Carnets de géographes [En ligne], 5 | 2013, mis en ligne le 01 janvier 2013, consulté le 24 septembre 2020. URL : http://journals.openedition.org/cdg/1111 ; DOl : https://doi.org/10.4000/ cdg. 1111

\section{(c) (i) (3)}

La revue Carnets de géographes est mise à disposition selon les termes de la Licence Creative Commons Attribution - Pas d'Utilisation Commerciale - Pas de Modification 4.0 International. 


\title{
MATÉRIALISATIONS DU SOUVENIR EN MONTAGNE LES ENJEUX IDENTITAIRES DES PLACES ET DES PLACEMENTS
}

\author{
EMMANUELLE PETIT
}

Cette thèse s'intéresse à un ensemble d'objets qui matérialisent différents types de souvenirs au sein des Alpes Occidentales. L'originalité de ces artefacts réside dans leur nature même : ils figurent tous l'idée de montagne sous diverses formes et pour différents motifs. Il s'agit tout aussi bien de monuments érigés au détour d'une rue ou sur une place centrale pour commémorer un exploit, une catastrophe ou l'œuvre d'un homme en relation avec la montagne, que de stèles funéraires, profilées à l'image de sommets, érigées ça et là dans les cimetières, ou encore de plaques scellées à même le roc de la montagne.

À partir d'une réflexion sur le façonnement de ces artefacts qui jouent avec la figure de la montagne, cette recherche interroge le rôle de l'espace dans les processus mémoriels et identitaires. Elle propose une lecture interobjective par l'identification, la spatialisation et la généalogie des différentes manières de mettre en scène le souvenir au sein des Alpes Occidentales. Elle aborde également à partir de récits produits dans deux contextes spécifiques (Bessans en Haute-Maurienne (Savoie), Chamonix en Haute-Arve (Haute-Savoie)), selon une approche intersubjective cette fois, les relations que les hommes nouent avec ces artefacts, qu'ils vivent quotidiennement au contact de ces derniers ou qu'ils les contemplent de manière tout à fait occasionnelle. Cette démarche et ce terrain permettent de dégager les enjeux identitaires de la mise en visibilité des souvenirs et de souligner le rôle de l'espace dans ces processus.

Cette thèse défend l'idée que les artefacts sont centraux dans l'établissement des rapports sociaux. Ils participent à la construction des mondes de chacun et jouent un rôle actif dans les relations à soi et à l'autre autour d'un ensemble de jeux d'échelles et de métriques. Les artefacts du souvenir seraient donc à la fois un ferment et un révélateur du fonctionnement identitaire de la société.

Cette recherche repose à la fois sur un recensement et une description des figurations de la montagne concernant différents types d'artefacts du souvenir, sur un travail d'archives reconstituant leur généalogie et enfin sur une soixantaine d'entretiens « focalisés » avec des acteurs institutionnels, des habitants des communes enquêtées et des touristes. Elle a aussi bénéficié des apports d'une recherche-action menée parallèlement dans le contexte chamoniard. 
Elle se déroule en sept chapitres.

Le premier chapitre pose les fondements conceptuels nécessaires à l'appréhension des artefacts érigés pour se souvenir en des lieux spécifiques et des constructions qui prennent forme à leur contact. Ce cheminement théorique, construit en confrontation avec le terrain, a pris forme autour de plusieurs nécessités. La première nécessité est conceptuelle : expliciter la conception de l'objet défendue dans cette démarche et l'intérêt du recours au terme d'artefact en définissant le rôle social attribué aux objets. La seconde nécessité relève du l'ordre du disciplinaire. Mener une recherche sur des objets fabriqués pour se souvenir n'est pas une thématique habituelle en géographie. Aussi est-il apparu incontournable de positionner cette recherche dans le champ disciplinaire tout en exposant les apports de l'ensemble des sciences sociales et en dégageant les enjeux spatiaux autour de la mémoire, de l'identité et des objets les matérialisant. Le premier temps de ce premier chapitre témoigne de l'ancrage des objets dans la discipline. La géographie s'intéresse à eux dès ses débuts institutionnels avec une conception extrêmement matérialiste. Les objets sont considérés comme des témoins permettant de prouver la véracité de l'analyse. Abandonnés dans les années 1960 au profit soit d'une géographie quantitative soit au profit d'une géographie humaniste tournée sur le sujet, les objets peuvent retrouver leur place dans la discipline grâce aux apports de l'ensemble des sciences humaines et sociales. Le deuxième temps invite à passer de l'objet témoin figé d'une histoire à l'objet social actif dans les configurations sociales et les constructions identitaires dont le sens est sans cesse renégocié, toujours ou parfois cristallisé, sédimenté justement. Cette conception est un moyen de renouveler les recherches autour de ce que certains nomment la culture matérielle. Un dernier temps aborde enfin la spécificité mémorielle des objets concernés, leur incontournable dimension spatiale et identitaire, et justifie l'emploi du terme d'artefact du souvenir comme un moyen de ne pas enfermer par avance les objets dans des catégories déterminées a priori, par avance.

Le second chapitre propose une scénarisation du dispositif méthodologique. Scénarisation dans le sens où cette présentation méthodologique est a posteriori une mise en cohérence d'une pratique du terrain qui s'est effectuée à tâtons par des allers et retours incessants entre observations, inductions, hypothèses, déductions. Au fil des découvertes, les attendus ont parfois changé de forme, modifiant en retour le fil de la recherche. Il insiste sur la nécessité de croiser un ensemble de techniques pour cerner toutes les dimensions de l'artefact et appréhender les constructions/déconstructions/reconstructions dont ils font l'objet. Ainsi, de manière imbriquée, observations, entretiens, recherche-action, et recherche documentaire ont donné lieu à des moments de terrain bien distincts. Ce chapitre vise aussi à rendre accessible la subjectivité du chercheur active sur le terrain, en concevant cette mise à jour comme un moyen de maitriser les cadres d'interprétations de cette recherche qualitative. C'est donc pour partie un retour réflexif sur le cheminement de cette thèse au fil des découvertes.

Les troisième et quatrième chapitres s'attèlent à l'une des facettes de l'identité en tentant d'exciper ce qui distingue ces artefacts, ce qui permet de le définir. L'artefact est dans ce cas analysé de façon objective. Les artefacts sont minutieusement décrits pour dresser leurs caractéristiques identitaires respectives. Où se situent-ils ? Quand et pourquoi ont-ils été érigés? À quelles mises en scène a-t-on affaire? À qui ces mises en scène sont-elles destinées ? Sur quels attributs la figuration de la montagne est-elle construite ? Répondre à ces questions a permis d'établir en quelque sorte une carte d'identité et de dégager les différentes façons d'afficher une singularité ou une appartenance. Cette forme de description 
ethnographique de l'artefact est une mise en évidence des différentes rhétoriques spatiales identitaires mises en place, dans chacun des contextes socio-culturels étudiés, pour se différencier et/ou se reconnaître membre d'un collectif plus ou moins vaste. Cette description se déroule en deux temps et avec deux types d'approche. Le troisième chapitre propose une analyse morphologique des artefacts à partir de décomptes statistiques. Il s'intéresse plus particulièrement à la question de la localisation et à l'identification de différentes manières de mettre en scène le souvenir, d'où résulte une spatialisation de différentes formes de mise en visibilité du souvenir. Le quatrième chapitre traite quant à lui de la question des temporalités d'édification des artefacts et des acteurs en jeu. Il propose une reconstitution généalogique des différentes manières de faire, tel un récit inaugural sur lequel et avec lequel les constructions identitaires prennent aujourd'hui forme.

Les cinquième, sixième et septième chapitres reconsidèrent la question de l'identité à partir de l'artefact, en ne l'interrogeant non plus à partir de l'objet même, mais à partir de la relation que les individus nouent avec ces objets fabriqués. Ils permettent ainsi de montrer que cette relation est aussi créatrice d'identité, que l'artefact ainsi que l'emplacement qu'il occupe font sens pour ceux qui le pratiquent. Au travers de l'analyse des récits, ces chapitres révèlent tantôt comment l'artefact est un moyen pour les individus de définir leur face d'acteur social inséré dans un collectif, tantôt un moyen de se distinguer de l'autre. L'artefact est cette fois envisagé de façon intersubjective puisqu'il s'agit, non pas d'établir l'état descriptif des significations produites par les différents acteurs concernant les mises en scène du souvenir, mais bien de mettre en évidence comment leurs récits construisent et reposent sur différents dispositifs, différents agencements spatiaux du souvenir qui règlent pour partie les constructions identitaires. Les cinquième et sixième chapitres s'intéressent tout particulièrement aux questions d'emplacement et de place, alors que le septième chapitre s'attèle à l'importance de la mise en scène quant à l'attribution de la place.

$\mathrm{Au}$ bout du compte, cette thèse tente de prouver qu'à partir d'un tout petit objet, il est possible de saisir de nombreux enjeux du fonctionnement de la vie en société, qui s'expriment entre autres par la recherche, l'octroi, la tenue, la défense d'une place. Elle constitue en cela une invitation au développement d'une micro-géographie attentive aux individus, à ce qu'ils disent, à ce qu'ils font, et à ce qui légitime leur place, celle qu'ils veulent tenir et celle qu'on leur fait tenir, à travers les rapports sociaux qui se nouent et se dénouent autour de ces artefacts. 
Fiche informative

\section{Lien électronique}

http://tel.archives-ouvertes.fr/tel-00752857

\section{Discipline}

Géographie

\section{Directeur}

Guy Di Méo

\section{Université}

Université Bordeaux 3

Membres du jury de thèse, soutenue le 28 septembre 2012

- Vincent Veschambre, Professeur de géographie, École Nationale d'Architecture de Lyon (Rapporteur) ;

- Michel Lussault, Professeur de géographie, Université de Lyon, Ecole Normale Supérieure de Lyon (Rapporteur) ;

- Patrick Baudry, Professeur de sociologie, Université Bordeaux 3 Michel de Montaigne (Examinateur);

- Béatrice Collignon, Maître de conférence de géographie, Université Paris 1 PanthéonSorbonne (Examinatrice) ;

- Isabelle Sacareau, Professeure de géographie, Université Bordeaux 3 Michel de Montaigne, (Présidente du jury)

\section{Situation professionnelle actuelle}

Membre associé de l'UMR ADES 5185 CNRS

\section{Courriel de l'auteur}

mailto:emmanuellepetit@1aposte.net 